PROCEEDINGS OF THE

AMERICAN MATHEMATICAL SOCIETY

Volume 134, Number 9, September 2006, Pages 2739-2744

S 0002-9939(06)08296-7

Article electronically published on April 10, 2006

\title{
CONVERGENCE OF WEIGHTED AVERAGES OF RANDOM VARIABLES REVISITED
}

\author{
NASROLLAH ETEMADI \\ (Communicated by Richard C. Bradley)
}

\begin{abstract}
We show that for a large class of positive weights including the ones that are eventually monotone decreasing and those that are eventually monotone increasing but vary regularly, if the averages of random variables converge in some sense, then their corresponding weighted averages also converge in the same sense. We will also replace the sufficient conditions in the fundamental result of Jamison, Pruitt, and Orey for i.i.d. random variables that make their work more transparent.
\end{abstract}

Let $\left\{X_{i}: i \geq 1\right\}$ be an arbitrary sequence of random variables and let the weights $\left\{w_{i}: i \geq 1\right\}$ be a sequence of positive numbers. Define the total weight by $W_{n}=\sum_{i=1}^{n} w_{i}$ and the weighted sum by $S_{n}=\sum_{i=1}^{n} w_{i} X_{i}$. It is desirable to know under what conditions on the weights and/or the finite-dimensional distributions of the underlying random variables the weighted averages $S_{n} / W_{n}, n \geq 1$, converge in some sense. Since we already have theorems concerning the convergence of regular averages, $\sum_{i=1}^{n} X_{i} / n, n \geq 1$, it is natural to ask for conditions on the weights which are decoupled from the random variables so that the convergence of weighted averages would follow in the same sense. Surprisingly, a large class of weights, including the ones that are monotone decreasing and monotone increasing but varying regularly (see Feller [5], p. 447), satisfy our requirement. Also, since for i.i.d. random variables the condition $\mathbf{E} X_{1}=0$ is both necessary and sufficient for the strong law, $\sum_{i=1}^{n} X_{i} / n \stackrel{\text { a.e. }}{\longrightarrow} 0$, it is also desirable to know for what specific weights the almost everywhere convergence of the weighted averages would ensure that $\mathbf{E} X_{1}=0$.

A benchmark for convergence of weighted averages of i.i.d. random variables is the work of Jamison, Orey, and Pruitt [9], where in their fundamental Theorem 2 , p. 41, they have sufficient conditions on the marginal distribution of $X_{1}$ and on the weights so that the weighted average converges almost everywhere. In this note, first we answer some of the questions raised above and then replace their sufficient conditions by ones that make their work much more transparent. For another equivalent condition in terms of an infinite series, see 8, and for more on the necessity of these conditions, see Chen, X. et al. 11. Finally, see Liang [1] and the references there for further development of this subject.

Received by the editors December 6, 2004 and, in revised form, March 31, 2005 and April 14, 2005.

2000 Mathematics Subject Classification. Primary 60F15.

Key words and phrases. Weighted averages, limit theorems, pairwise independence.

This work was partially supported by the Mahani Mathematical Research Center. 
Lemma 1. Let $\left\{a_{i}: i \geq 1\right\}$ and $\left\{b_{i}: i \geq 1\right\}$ be two sequences of positive real numbers such that $A_{n}=\sum_{i=1}^{n} a_{i} \rightarrow \infty, B_{n}=\sum_{i=1}^{n} b_{i} \rightarrow \infty$, and

$$
\text { (1) } \sup _{n \geq 1}\left(\frac{b_{n} A_{n}}{a_{n} B_{n}}\right)<\infty \quad \& \quad \text { (2) } \sup _{n \geq 2} \sum_{i=1}^{n-1}\left(\frac{\left|\frac{b_{i}}{a_{i}}-\frac{b_{i+1}}{a_{i+1}}\right| A_{i}}{B_{n}}\right)<\infty .
$$

Let $\left\{x_{i}: i \geq 0\right\}$ be another sequence of real numbers. Then

$$
\frac{\sum_{i=1}^{n} a_{i} x_{i}}{A_{n}} \rightarrow x_{0} \quad \Rightarrow \quad \frac{\sum_{i=1}^{n} b_{i} x_{i}}{B_{n}} \rightarrow x_{0} .
$$

Furthermore, condition (2) is not needed if the sequence $\left\{b_{i} / a_{i}: i \geq 1\right\}$ is monotone increasing, and neither one is needed when the sequence $\left\{b_{i} / a_{i}: i \geq 1\right\}$ is monotone decreasing.

Proof. Let $y_{n}=\sum_{i=1}^{n} a_{i} x_{i} / A_{n}$. By centering $x_{i}$ 's at $x_{0}$ without loss of generality, we may assume $y_{n} \rightarrow 0$. Now an easy manipulation shows that for $n \geq 2$,

$$
\frac{\sum_{i=1}^{n} b_{i} x_{i}}{B_{n}}=\frac{\frac{b_{n}}{a_{n}} A_{n} y_{n}+\sum_{i=1}^{n-1}\left(\frac{b_{i}}{a_{i}}-\frac{b_{i+1}}{a_{i+1}}\right) A_{i} y_{i}}{B_{n}} .
$$

Since our assumption trivially justifies the conditions of a Toeplitz's theorem (see Knopp [10, Theorem 4 on page 74), we are done with our first assertion. To see the second one let us consider the case where $b_{i} / a_{i}$ is monotone increasing. In this case,

$$
\begin{aligned}
\sum_{i=1}^{n-1}\left(\left|\frac{b_{i}}{a_{i}}-\frac{b_{i+1}}{a_{i+1}}\right| A_{i}\right) & =\sum_{i=1}^{n-1}\left(\left(\frac{b_{i+1}}{a_{i+1}}-\frac{b_{i}}{a_{i}}\right) A_{i}\right) \leq \sum_{i=1}^{n-1}\left(\frac{b_{i+1}}{a_{i+1}} A_{i+1}-\frac{b_{i}}{a_{i}} A_{i}\right) \\
& =\frac{b_{n}}{a_{n}} A_{n}-\frac{b_{1}}{a_{1}} A_{1} \leq \frac{b_{n}}{a_{n}} A_{n},
\end{aligned}
$$

which gives us the same bound as in condition (1). On the other hand assume $b_{i} / a_{i}$ is monotone decreasing. Then

$$
\begin{aligned}
\sum_{i=1}^{n-1}\left(\left|\frac{b_{i}}{a_{i}}-\frac{b_{i+1}}{a_{i+1}}\right| A_{i}\right) & =\sum_{i=1}^{n-1}\left(\frac{b_{i}}{a_{i}} A_{i}-\frac{b_{i+1}}{a_{i+1}} A_{i+1}\right)+\sum_{i=1}^{n-1}\left(\frac{b_{i+1}}{a_{i+1}}\left(A_{i+1}-A_{i}\right)\right) \\
& =\frac{b_{1}}{a_{1}} A_{1}-\frac{b_{n}}{a_{n}} A_{n}+\sum_{i=1}^{n-1} b_{i+1} .
\end{aligned}
$$

Thus (1) and (2) are bounded by

$$
\sup _{n \geq 1}\left(\frac{b_{n} A_{n}}{a_{n} B_{n}}+\sum_{i=1}^{n-1}\left(\frac{\left|\frac{b_{i}}{a_{i}}-\frac{b_{i+1}}{a_{i+1}}\right| A_{i}}{B_{n}}\right)\right)=\sup _{n \geq 1}\left(\frac{\frac{b_{1}}{a_{1}} A_{1}+B_{n}-B_{1}}{B_{n}}\right)<\infty,
$$

and we have the desired result.

The non-probabilistic version of the last assertion in the following theorem can also be found in Knopp [10]; see Exercise 30 on page 109.

Theorem 1. Let $\left\{w_{i}: i \geq 1\right\}$ be a sequence of positive weights such that $W_{n}=$ $\sum_{i=1}^{n} w_{i} \rightarrow \infty$. Let

$$
\text { (1) } \sup _{n \geq 1} \frac{n w_{n}}{W_{n}}<\infty \quad \& \quad \text { (2) } \sup _{n \geq 2} \sum_{i=1}^{n-1}\left(\frac{i\left|w_{i+1}-w_{i}\right|}{W_{n}}\right)<\infty .
$$


Let $\left\{X_{i}: i \geq 0\right\}$ be a sequence of random variables. Then

$$
\begin{gathered}
\frac{1}{n} \sum_{i=1}^{n} X_{i} \stackrel{\mathbf{P}}{\longrightarrow} X_{0} \Rightarrow \frac{1}{W_{n}} \sum_{i=1}^{n} w_{i} X_{i} \stackrel{\mathbf{P}}{\longrightarrow} X_{0} \quad \& \\
\frac{1}{n} \sum_{i=1}^{n} X_{i} \stackrel{\text { a.e. }}{\longrightarrow} X_{0} \Rightarrow \frac{1}{W_{n}} \sum_{i=1}^{n} w_{i} X_{i} \stackrel{\text { a.e. }}{\longrightarrow} X_{0},
\end{gathered}
$$

and if $\left\{\left|X_{i}\right|^{p}: i \geq 1\right\}, p \geq 1$, is uniformly integrable, then

$$
\frac{1}{n} \sum_{i=1}^{n} X_{i} \stackrel{\mathbf{L}^{p}}{\longrightarrow} X_{0} \Rightarrow \frac{1}{W_{n}} \sum_{i=1}^{n} w_{i} X_{i} \stackrel{\mathbf{L}^{p}}{\longrightarrow} X_{0} .
$$

Furthermore, condition (2) is not needed if the sequence $\left\{w_{i}: i \geq 1\right\}$ is monotone increasing, and both (1) and (2) are not needed if the sequence is monotone decreasing.

Proof. In light of Lemma 1, the almost everywhere convergence is immediate, and convergence in probability is an obvious consequence of the fact that, in general, a sequence of random variables converges in probability iff every subsequence has a further subsequence that converges almost everywhere. Finally since $\mathbf{L}^{p_{-}}$ convergence implies convergence in probability, we only need to show that the sequence $\left\{S_{n}:=\sum_{i=1}^{n} w_{i}\left(X_{i}-X_{0}\right) / W_{n}: n \geq 1\right\}$ is uniformly integrable. To this end, let $M>0$ and $p \geq 1$, by Jensen's inequality, and then breaking up the set $\left\{\left|\frac{S_{n}}{W_{n}}\right|>M^{1 / p}\right\}$ over the set $\left\{\left|X_{i}-X_{0}\right|>M^{1 / p}\right\}$ and its complement, we obtain

$$
\begin{aligned}
\mathbf{E}\left(\left|\frac{S_{n}}{W_{n}}\right|^{p} I\left\{\left|\frac{S_{n}}{W_{n}}\right|^{p}>M\right\}\right) \leq & \frac{1}{W_{n}} \sum_{i=1}^{n} w_{i} \mathbf{E}\left(\left|X_{i}-X_{0}\right|^{p} I\left\{\left|\frac{S_{n}}{W_{n}}\right|>M^{1 / p}\right\}\right) \\
\leq & \sup _{i \geq 1} \mathbf{E}\left(\left|X_{i}-X_{0}\right|^{p} I\left\{\left|X_{i}-X_{0}\right|>M^{1 / p}\right\}\right) \\
& +M \mathbf{P}\left\{\left|\frac{S_{n}}{W_{n}}\right|>M^{1 / p}\right\} .
\end{aligned}
$$

But $\sum_{i=1}^{n} X_{i} / n \stackrel{\mathbf{L}^{p}}{\longrightarrow} X_{0}$ implies that $X_{0} \in \mathbf{L}^{p}$. Therefore by our assumption $\left\{\left|X_{i}-X_{0}\right|^{p}: i \geq 1\right\}$ is uniformly integrable, and this is all we need to let $M \rightarrow \infty$ to conclude the theorem.

Next we will prove the fundamental theorem in Jamison, et al. 9], but for pairwise independent random variables, and then we will show that our conditions are equal to theirs. In extending their result to the pairwise independent case we had only pointed out, in Etemadi [4], that these results follow from the following version of Kolomogorov's theorem. However, to show that the conditions in our version (given as Theorem 3) are the same as theirs, and since the proof is short, we have provided the complete proof.

Theorem 2 (Etemadi [4). Let $\left\{X_{i}: i \geq 1\right\}$ be a sequence of pairwise independent random variables. Let $\left\{w_{i}: i \geq 1\right\}$ be a set of positive weights such that $W_{n}=$ $\sum_{i=1}^{n} w_{i} \rightarrow \infty$ and $w_{n} / W_{n} \rightarrow 0$. Then

$$
\begin{gathered}
\text { (1) } \sup _{n \geq 1} \mathbf{E}\left|X_{n}-\mathbf{E} X_{n}\right|<\infty \quad \& \\
\text { (2) } \sum_{n=1}^{\infty} \frac{w_{n}^{2} \mathbf{V a r} X_{n}}{W_{n}^{2}}<\infty \Rightarrow \frac{\sum_{i=1}^{n} w_{i}\left(X_{i}-\mathbf{E} X_{i}\right)}{W_{n}} \stackrel{\text { a.e. }}{\longrightarrow} 0 .
\end{gathered}
$$


Remark. For a weakening of condition (1) and the fact that condition (2) alone is not sufficient for their conclusion to hold, see Csörgö et al. [2, for a practical example of pairwise independent but not mutually independent random variables, see Geisser and Mantel [6], for how important pairwise independent random variables are, see O'Brein [12] and Harber 7], and finally for a survey of their application in computer science, see Wigderson [13].

Theorem 3. Let $\left\{X_{i}: i \geq 1\right\}$ be a sequence of pairwise independent, identically distributed random variables such that $\mathbf{E}\left|X_{1}\right|<\infty$. Let $\left\{w_{i}: i \geq 1\right\}$ be a set of positive weights such that $W_{n}=\sum_{i=1}^{n} w_{i} \rightarrow \infty$ and $w_{n} / W_{n} \rightarrow 0$. Let $N(x)=$ $\#\left\{n: W_{n} / w_{n} \leq x\right\}$ be the distribution function of $\left\{W_{n} / w_{n}: n \geq 1\right\}$. Then

$$
\int_{0}^{1} x \mathbf{E} N\left(\frac{\left|X_{1}\right|}{x}\right) d x<\infty \Rightarrow \frac{1}{W_{n}} \sum_{i=1}^{n} w_{i} X_{i} \stackrel{\text { a.e. }}{\longrightarrow} \mathbf{E} X_{1} .
$$

Proof. Since $\sup _{n \geq 1} \mathbf{E}\left|X_{n}-\mathbf{E} X_{n}\right| \leq 2 \mathbf{E}\left|X_{1}\right|<\infty$ and truncated pairwise independent random variables remain pairwise, due to Kolmogorov's condition (2) of Theorem 2, the proof will be no different than the classical strong law; see Jamison et al. [9]. It amounts to showing that

$$
\sum_{n=1}^{\infty} \mathbf{P}\left\{\left|X_{n}\right| \geq \frac{W_{n}}{w_{n}}\right\}<\infty \text { and } \sum_{n=1}^{\infty} \frac{w_{n}^{2}}{W_{n}^{2}} \mathbf{E}\left(\left|X_{n}\right|^{2} I\left\{\left|X_{n}\right|<\frac{W_{n}}{w_{n}}\right\}\right)<\infty .
$$

Since the random variables are identically distributed, and for any random variable $X$ and $T>0$,

$$
\begin{aligned}
\frac{1}{T^{2}} \mathbf{E}\left(|X|^{2} I\{|X|<T\}\right)+\mathbf{P}\{|X| \geq T\} & =\frac{2}{T^{2}} \int_{[0, T)} x \mathbf{P}\{|X| \geq x\} d x \\
& =2 \int_{0}^{1} x \mathbf{P}\{|X| \geq T x\} d x,
\end{aligned}
$$

the sum of the above two series can be written as

$$
\begin{aligned}
\sum_{n=1}^{\infty} & \left(\frac{w_{n}^{2}}{W_{n}^{2}} \mathbf{E}\left(\left|X_{1}\right|^{2} I\left\{\left|X_{1}\right|<\frac{W_{n}}{w_{n}}\right\}\right)+\mathbf{P}\left\{\left|X_{1}\right| \geq \frac{W_{n}}{w_{n}}\right\}\right) \\
& =2 \sum_{n=1}^{\infty} \int_{0}^{1} x \mathbf{P}\left\{\left|X_{1}\right| \geq \frac{W_{n}}{w_{n}} \cdot x\right\} d x \\
& =2 \int_{0}^{1} x \sum_{n=1}^{\infty} \mathbf{P}\left\{\left|X_{1}\right| \geq \frac{W_{n}}{w_{n}} \cdot x\right\} d x \\
& =2 \int_{0}^{1} x \mathbf{E} N\left(\frac{\left|X_{1}\right|}{x}\right) d x<\infty .
\end{aligned}
$$

Next we show that our conditions are the same as the ones given in Theorem 2 of Jamison et al. [9].

Lemma 2. Under the same setup as in Theorem 3,

$$
\int x^{2} \int_{y>|x|} \frac{N(y)}{y^{3}} d y d F(x)=\int_{0}^{1} x \mathbf{E} N\left(\frac{\left|X_{1}\right|}{x}\right) d x,
$$

where $F(x)=\mathbf{P}\left\{X_{1} \leq x\right\}$ is the probability distribution function of $X_{1}$. 
Proof. By Tonelli's theorem, for $x \in(-\infty, \infty)$,

$$
\begin{aligned}
\int_{(|x|, \infty)} \frac{d N(u)}{u^{2}} & =2 \int_{(|x|, \infty)}\left(\int_{[u, \infty)} \frac{d y}{y^{3}}\right) d N(u)=2 \int_{(|x|, \infty)} \frac{1}{y^{3}} \int_{(|x|, y]} d N(u) d y \\
& =2 \int_{(|x|, \infty)} \frac{1}{y^{3}}(N(y)-N(|x|)) d y=2 \int_{(|x|, \infty)} \frac{N(y)}{y^{3}} d y-\frac{N(|x|)}{x^{2}} .
\end{aligned}
$$

Hence,

$$
\begin{aligned}
& \sum_{n=1}^{\infty} \frac{w_{n}^{2}}{W_{n}^{2}} \mathbf{E}\left(\left|X_{n}\right|^{2} I\left\{\left|X_{n}\right|<\frac{W_{n}}{w_{n}}\right\}\right) \\
& =\mathbf{E}\left(X_{1}^{2} \sum_{\left\{n: \frac{W_{n}}{w_{n}}>\left|X_{1}\right|\right\}} \frac{w_{n}^{2}}{W_{n}^{2}}\right)=\mathbf{E}\left(X_{1}^{2} \int_{\left(\left|X_{1}\right|, \infty\right)} \frac{d N(u)}{u^{2}}\right) \\
& =\mathbf{E}\left(2 X_{1}^{2} \int_{\left(\left|X_{1}\right|, \infty\right)} \frac{N(y)}{y^{3}} d y-N\left(\left|X_{1}\right|\right)\right) .
\end{aligned}
$$

But,

$$
\mathbf{E} N\left(\left|X_{1}\right|\right)=\mathbf{E}\left(\sum_{n=1}^{\infty} I\left\{\frac{W_{n}}{w_{n}} \leq\left|X_{1}\right|\right\}\right)=\sum_{n=1}^{\infty} \mathbf{P}\left\{\left|X_{1}\right| \geq \frac{W_{n}}{w_{n}}\right\} .
$$

As a result

$$
\begin{gathered}
\sum_{n=1}^{\infty}\left(\frac{w_{n}^{2}}{W_{n}^{2}} \mathbf{E}\left(\left|X_{n}\right|^{2} I\left\{\left|X_{n}\right|<\frac{W_{n}}{w_{n}}\right\}\right)+\mathbf{P}\left\{\left|X_{1}\right| \geq \frac{W_{n}}{w_{n}}\right\}\right) \\
=2 \int x^{2} \int_{y>|x|} \frac{N(y)}{y^{3}} d y d F(x),
\end{gathered}
$$

where the left-hand side is the same as the one at the bottom of Theorem 3 .

Finally, we give the following corollary as another example of how Theorem 1 can be utilized.

Corollary. Use the same setup as in Theorem 3. If the weights are monotone increasing, and the weighted averages converges to zero almost everywhere, then $\mathbf{E} X_{1}=0$.

Proof. The proof is an immediate consequence of Theorem 1 and the fact that the necessity condition for the strong law for i.i.d. random variables remains intact for pairwise i.i.d. as well; see Etemadi [3].

\section{ACKNOWLEDGMENT}

I am grateful to Professor Mehdi Radjabalipour for his unyielding support during my stay at the Mahani Mathematical Research Center.

\section{REFERENCES}

1. Chen, X., Zhu, L. X., Fang, K.T. (1996), Almost sure convergence of weighted sum. Statistical Sinica, 6, 499-507. MR1399318 (97f:60067)

2. Csörgö, S., Tandori, K., and Totik, V. (1983), On the strong law of large numbers for pairwise independent random variables, Acta Math. Hung. 42, 319-330. MR 0722846 (85e:60034)

3. Etemadi, N. (1981), An elementary proof of the strong law of large numbers, Z Warrsch. Gebiete 55, 119-122. MR0606010(82b:60027) 
4. Etemadi, N. (1983), Stability of Sums of Weighted Nonnegative Random Variables, Journal of Multivariate Analysis, Vol. 13, No. 2, 361-365. MR0705557 (85a:60035)

5. Feller, W. (1971), An Introduction to Probability Theory and Its Applications, Volume II, 2nd Ed., John Wiley \& Sons Inc., New York. MR0270403 (42:5292)

6. Geisser, S. and Mantel, N. (1962), Pairwise independence of jointly dependent variables, The Annals of Statistics, Vol. 33, No. 1, 290-291. MR0137188 (25:644)

7. Harber, M. (1986), Testing for pairwise independence, Biometrics 42, 429-435.

8. Heyde, C. C. (1968), On almost sure convergence for sums of independent random variables, Sankhya A 30, 353-358. MR.0279865 (43:5586)

9. Jamison, B., Orey, S., and Pruitt, W. (1965), Convergence of weighted averages of independent random variables, Z. Wahrsch. Gebiete 4, 40-44. MR0182044 (31:6268)

10. Knopp, K. (1990), Theory and Application of Infinite Series. Dover Publishing Company. MR.0028430 (10:446a)

11. Liang, H. Y. (2000), Complete convergence for weighted sums of negatively associated random variables, Statistics \& Probability Letters, 48, 317-325. MR.1771494(2001e:60064)

12. O'Brein G. L. (1980), Pairwise independent random variables, The Annals of Probability, Vol. 8, No. 1, 170-175. MR0556424 (81d:60011)

13. Wigderson, A. (1994), The amazing power of pairwise independence. Proceedings of the twenty-sixth annual ACM symposium on Theory of Computing, Stoc. 645-647, Montreal, Canada.

Department of Mathematics, Statistics, \& Computer Science, University of Illinois at Chicago, 322 Science \& Engineering Offices (SEO) m/C 249, 851 S. Morgan Street, Chicago, Illinois 60607-7045

E-mail address: Etemadi@uic.edu 